\title{
An advanced glioma cell invasion assay based on organotypic brain slice cultures
}

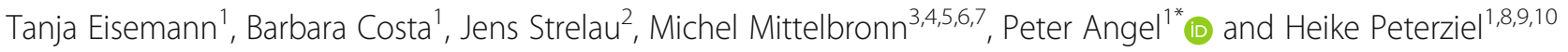

\begin{abstract}
Background: The poor prognosis for glioblastoma patients is caused by the diffuse infiltrative growth pattern of the tumor. Therefore, the molecular and cellular processes underlying cell migration continue to be a major focus of glioblastoma research. Emerging evidence supports the concept that the tumor microenvironment has a profound influence on the functional properties of tumor cells. Accordingly, substantial effort must be devoted to move from traditional two-dimensional migration assays to three-dimensional systems that more faithfully recapitulate the complex in vivo tumor microenvironment.
\end{abstract}

Methods: In order to mimic the tumor microenvironment of adult gliomas, we used adult organotypic brain slices as an invasion matrix for implanted, fluorescently labeled tumor spheroids. Cell invasion was imaged by confocal or epi-fluorescence microscopy and quantified by determining the average cumulative sprout length per spheroid. The tumor microenvironment was manipulated by treatment of the slice with small molecule inhibitors or using different genetically engineered mouse models as donors.

Results: Both epi-fluorescence and confocal microscopy were applied to precisely quantify cell invasion in this ex vivo approach. Usage of a red-emitting membrane dye in addition to tissue clearing drastically improved epifluorescence imaging. Preparation of brain slices from of a genetically engineered mouse with a loss of a specific cell surface protein resulted in significantly impaired tumor cell invasion. Furthermore, jasplakinolide treatment of either tumor cells or brain slice significantly reduced tumor cell invasion.

Conclusion: We present an optimized invasion assay that closely reflects in vivo invasion by the implantation of glioma cells into organotypic adult brain slice cultures with a preserved cytoarchitecture. The diversity of applications including manipulation of the tumor cells as well as the microenvironment, permits the investigation of rate limiting factors of cell migration in a reliable context. This model will be a valuable tool for the discovery of the molecular mechanisms underlying glioma cell invasion and, ultimately, the development of novel therapeutic strategies.

Keywords: migration, organotypic brain slices, tumor microenvironment, glioblastoma, three-dimensional invasion assay

\section{Background}

Glioblastoma is the most frequent and malignant primary brain tumor, with a median survival of 12-15 months after diagnosis. Despite extensive surgical resection, chemo-, and radiotherapy, glioblastoma is still considered incurable [1-3]. The diffuse infiltration of tumor cells into adjacent healthy brain tissue is a major cause of treatment failure, and so the characterization of signaling pathways and effector molecules that drive glioblastoma invasion is a major aim in glioblastoma research (for reviews see $[4,5]$ ).

\footnotetext{
*Correspondence: p.angel@dkfz.de

'Division of Signal Transduction and Growth Control, DKFZ/ZMBH Alliance,

Heidelberg, Germany

Full list of author information is available at the end of the article
}

Most studies of tumor cell migration involve simple and inexpensive two-dimensional methods like the in vitro scratch and Boyden chamber/transwell assays. However, recent studies have shown striking differences in protein functions in two- and three-dimensional contexts [6-8]. Furthermore, in vivo tumor cells are embedded in a three-dimensional matrix consisting of the extracellular matrix (ECM) and multiple cell types, which can all interact with tumor cells. Emerging evidence highlights the substantial impact of these reciprocal interactions within the tumor microenvironment on tumor cell invasion [9], and therefore the requirement for an invasion assay that closely mimics the environmental milieu 
that glioma cells encounter in vivo. Invading glioblastoma cells follow distinct anatomical features called Scherer's structures. These include meninges and the subjacent subarachnoid space, blood vessels, myelinated nerve fibers and the extracellular space between neuronal or glial processes in the brain parenchyma [10]. Taking into account that glioblastoma cells migrate along these pre-existing multicellular structures - that cannot simply be mimicked by co-cultivation of the relevant cell types - we used organotypic murine brain slice cultures as a three-dimensional invasion matrix. Preserving essential features of the host tissue such as neuronal connectivity, glial-neuronal interactions and an authentic ECM, organotypic brain slice cultures have mainly been used to study developmental, structural and electrophysiological aspects of neuronal circuits (for reviews see [11, 12]). Previously, these organotypic cultures have also been presented as a novel tool to examine the migratory behavior of ex vivo implanted tumor cells [13-16]. However, the reported methods were based on human brain slices, or the extent of invasion observed was rather low and did not reflect the high infiltration capacity of glioblastoma cells in vivo. Here, we present an optimized and reproducible protocol to assess highly infiltrating glioma cells in an adult murine brain slice. In particular, we show that the usage of a membrane dye with red-shifted fluorescence spectra and tissue clearing results in greatly increased image quality. Finally, we present a selection of application examples, including the treatment of tumor cells or the manipulation of the tumor cell environment by pharmacological inhibitors and the use of genetically modified mice as brain slice donors. Knowledge gained from in vitro and high-throughput approaches can be functionally validated by this method, accentuating its value as link between in vitro and animal studies.

\section{Methods}

\section{Preparation of brain slices}

6-8 week old $\mathrm{C} 57 \mathrm{Bl} / 6$ wild-type or knockout mice were euthanized, the brain was isolated and the cerebellum removed with a scalpel. Using insect forceps the brain was transferred to the vibratome (Leica VT1200 S) platform and immediately fixed to this device by applying a drop of superglue. The lateral short side of the brain was placed facing the blade, in order to reduce mechanical stress. $350 \mu \mathrm{m}$ thick coronal slices were cut with a maximal speed of $0.2 \mathrm{~mm} / \mathrm{s}$. Up to three slices were gathered per filter (Millipore \#PICM03050). The transfer of the slices was facilitated by a brush and addition of brain slice medium on top of the filter. The brain slice medium is composed of MEM (Sigma \# M2279), 25\% heatinactivated horse serum (Life Technologies \# 26050070), 25 mM HEPES (Sigma \# H0887-100 mL), 1 mM L- glutamine (Sigma, \# G7513), $5 \mathrm{mg} / \mathrm{ml}$ glucose (Sigma \#
G8769), $100 \mathrm{U} / \mathrm{ml}$ penicillin/streptomycin (Sigma \# P4333). For cultivation at $37{ }^{\circ} \mathrm{C}$ and $5 \% \mathrm{CO}_{2}$ the medium was removed from the filter and $1 \mathrm{ml}$ of fresh brain slice medium was added below. The medium was refreshed after 18-24 $\mathrm{h}$ and then every other day. Brain slices were cultivated air-exposed. To prevent dehydration the tissue was moistened with a drop of medium every day, and remaining excess medium removed. Although the brain slices can be cultivated for at least one week, experiments were performed at $\mathrm{d} 2$ and, due to the high migratory capacity of glioma cells, terminated on $\mathrm{d} 4$.

\section{Preparation of fluorescently labeled spheroids}

Murine (SMA560) and human (LN319; U87MG; U251MG) glioma cell lines cultivated in serum-containing medium (DMEM, Sigma \# D5671; 10\% FBS, Sigma \# F7524; 2 mM L-glutamine) were trypsinized and counted. $1 \times 10^{6}$ cells $/ \mathrm{ml}$ PBS were incubated with $5 \mu \mathrm{l}$ lipophilic dye DiD $(1 \mathrm{mg} / \mathrm{ml}$ in DMSO, Biotium \#60014) or $5 \mu$ l DiI (Biotium \# 30022) for $30 \mathrm{~min}$ at $37^{\circ} \mathrm{C}$. After two washing steps 500 cells/well were seeded in a flat-bottom 96-well plate coated with $50 \mu \mathrm{l}$ low melt agarose (Genaxxon \# M3049.0010; 1\% in PBS). SMA560 and U87MG spheroids were cultivated in serum-containing DMEM medium, whereas the spheroid formation of LN319 and U251MG required cultivation in serum-free neurobasal medium (Thermo Fisher Scientific \# 10888022) containing B27 supplement (Thermo Fisher Scientific \# 17504044), $20 \mathrm{ng} / \mathrm{ml}$ of both EGF (Promokine \# C-60170) and FGFb (Promokine \# C-60240), $2 \mu \mathrm{g} / \mathrm{ml}$ heparin sodium salt (Sigma \# H3149), 2 mM L-glutamine and $1 \%$ penicillin/streptomycin $(\mathrm{p} / \mathrm{s} ; 100 \mathrm{U} / \mathrm{ml})$.

Human and murine primary cells were cultivated as spheroids in serum-free medium. Primary human cells were kept in neurobasal medium, and primary murine cells were cultivated in DMEM/F12 (Thermo Fisher Scientific \# 21331020) medium containing N 2 supplement (Life Technologies \# 17502048), $20 \mathrm{ng} / \mathrm{ml}$ of both EGF and FGFb, $2 \mathrm{mM}$ L-glutamine and 1\% penicillin/streptomycin (100 U/ml). To generate spheroids for subsequent implantations, cells were labeled as described above and seeded in agarose-free U-bottom 96-well plates (500 cells/well; Greiner \# 650185).

Most glioma cell lines and primary cells we tested formed spheroids under the described conditions. However, in some cases the protocol might have to be adjusted to cultivation in neurobasal medium, or to the hanging drop culture protocol. The last cultivation technique requires medium supplementation with $20 \%$ methyl cellulose. The single cell suspension is pipetted in drops of $20 \mu \mathrm{l}$ on the inside of a dish which is then slowly inverted.

For generation of heterotypic spheroids, astrocytes were isolated from 3 day old neonatal mice. Briefly, brains were isolated and the meninges removed. Cortical 
tissue was ground on a $70 \mu \mathrm{m}$ cell strainer with a glass pestle. After three washes, cells were cultured in DMEM (10\% FBS, $2 \mathrm{mM}$ L-glutamine and 1\% p/s) on poly-lysinecoated culture vessels. After a cultivation period of at least 10 days astrocytes were used for co-implantation experiments. Astrocytes were labeled with DiI and co-cultivated as heterotypic multicellular spheroids with $\mathrm{DiD}$ labeled murine SMA560 glioma cells line in a ratio of 3:2 in agarose coated 96-well plates for two days.

Due to the different growth rates of the tumor cells used in the experiments and the difficulty of precisely measuring the number of cells in an established spheroid, we seeded a fixed number of 500 cells and implanted the tumor cell spheroids when they reached a diameter of approximately $150 \mu \mathrm{m}$.

The murine cell line SMA560 was provided by Prof. Michael Platten (Department of Neurology, University Heidelberg). The human cell lines LN319 and U251 were provided by Prof. Wolfgang Wick (University Hospital Heidelberg), and Prof. Michael Weller (Department of Neurology, University Zurich) provided the human cell line U87MG. Human and murine primary glioblastoma cultures were generated in our laboratory.

\section{Tumor cell and brain slice treatment with jasplakinolide} 500 DiD labeled SMA560 glioma cells were seeded in spheroid-forming conditions per well of a 96-well plate. $18 \mathrm{~h}$ prior to implantation, spheroids or brain slices were treated with $1 \mu \mathrm{M}$ jasplakinolide (Cayman \#11705) or DMSO (Sigma \# 41639-100ML). $24 \mathrm{~h}$ after implantation, brain slices were fixed and imaged by confocal microscopy.

Cell viability in vitro was measured with trypan blue staining (Sigma \# 93595-50ML).

\section{Spheroid implantation}

8-10 spheroids per brain slice were manually implanted using a blunt Hamilton syringe (701 N; $10 \mu \mathrm{l} ; 26 \mathrm{~s} / 51 / 3)$ and a binocular microscope. For this purpose, a single spheroid was aspirated in a maximum volume of $0.5 \mu \mathrm{l}$ and implanted by release of the total volume into the tissue. This procedure was repeated until $8-10$ spheroids were implanted along the cortex (see Fig. 1 for implantation sites). There are several factors that are essential for maximum invasion. Most important in this context is proper implantation of the spheroids. It might help to completely penetrate through the tissue to the filter to estimate its depth; however, it is crucial to not release the spheroid onto the filter. The spheroid must be implanted within the tissue, as release of the spheroid below or on top of the brain tissue will not result in tumor cell invasion but in proliferation or in some cases in collective migration along the tissue surface. Furthermore, tissue integrity is an essential factor for correct implantation. Dehydration of brain slices impedes penetration of the tissue with the needle tip, as the tissue surface becomes too rigid. Conversely, excessive immersion of the brain slice in medium results in tissue degeneration and disintegration upon penetration with the needle tip. Thus, we recommend moistening the brain slices every other day, followed by removal of excessive medium. If these instructions are carefully followed, a successful implantation rate of at least $80 \%$ can be achieved.

Following implantation, medium was refreshed and the slices cultivated at $37{ }^{\circ} \mathrm{C}$ and $5 \% \mathrm{CO} 2$. Experiments were terminated $2 \mathrm{~d}$ after implantation unless otherwise stated. For fixation brain slice medium was removed and $1-2 \mathrm{ml} 4 \%$ PFA added on top of the filter for $2 \mathrm{~h}$ at RT
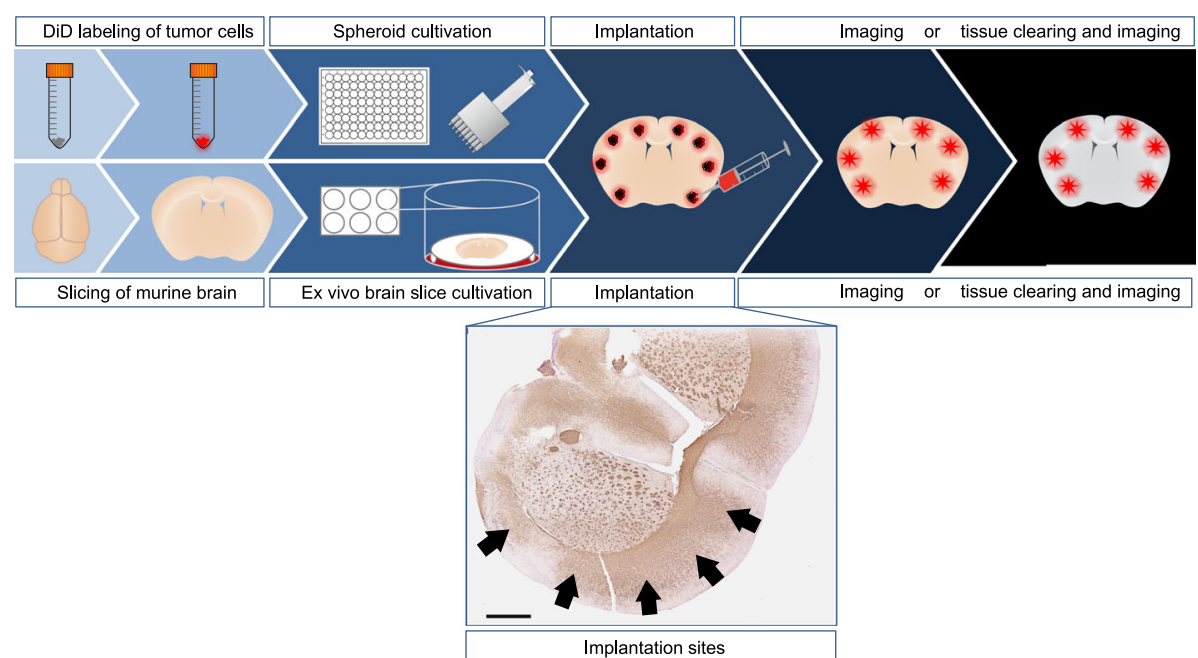

Fig. 1 Work flow of the ex vivo invasion assay based on organotypic brain slice cultures. Schematic representation of the individual protocol steps. Immunohistochemistry staining shows MBP pattern of an adult murine brain slice cultivated for four days, arrows indicate implantation sites, scale bar $1 \mathrm{~mm}$ 
or o/n at $4{ }^{\circ} \mathrm{C}$. Fixed slices were transferred with a spatula from the filter into a new 6-well plate containing $2 \mathrm{ml} \mathrm{PBS/well}$. Although the slices can be stored in the parafilm-sealed plate for at least three months in the dark at $4{ }^{\circ} \mathrm{C}$, slices were imaged by epi-fluorescence or confocal microscopy as soon as possible.

\section{Tissue clearing}

To obtain high quality epi-fluorescence microscopy images, autofluorescent brain slices were cleared according to the SeeDB protocol [17]. After incubation in $20 \%$ clearing solution for 4-8 $\mathrm{h}$ in the dark with gentle shaking, the clearing solution was changed to $40 \%$ and subsequently $60 \%$ clearing solution each for 4-8 h. Slices were sequentially incubated in $80 \%, 100 \%$ and $115 \%$ clearing solution $\mathrm{o} / \mathrm{n}$. As clearing solutions of $100 \%$ and $115 \%$ tend to crystalize, incubation steps were performed in a humid chamber. Cleared slices were stored in the $115 \%$ clearing solution in a dark humid chamber at RT. All clearing solutions contain 0,5\% $\alpha$-thioglycerol (Sigma \# M1753) and the above given wt/vol percentage of (D-) fructose (Sigma \# F0127).

\section{Imaging and quantification of invasion}

For epi-fluorescence imaging the slices were kept in 6-well plates containing PBS or, if the slices had been cleared, $115 \%$ clearing solution. For confocal imaging slices were transferred with a spatula onto an object slide and loosely covered with a coverslip.

Z-stack images were transformed to a maximum projection image by using ImageJ [18]. Image quality was optimized by adjusting brightness, contrast and gamma. Migratory cells were visible as spikes emerging from the bulk of the spheroids that had been formed by cells establishing an infiltration path. These invasion sprouts were traced from the center of the mass to the tip using the freehand tool. The radius of the spheroid body (if not determinable spheroid body radius from d0) was subtracted from the measured sprout length. Subsequently, we calculated the average cumulative sprout length by adding up the length of all sprouts of a spheroid and dividing this sum by the number of analyzed spheroids. This statistic integrates sprout length and the number of sprouts to estimate the migratory capacity of the cells. Calculation of the cumulative sprout length is a common tool in angiogenesis research, where it is used as reliable quantification of cell movement and proliferation in a three-dimensional environment [19-22].

\section{Statistical analysis}

Welch's t-test was performed to evaluate the difference between the cumulative sprout lengths of experimental groups. Differences in the grade of invasion were considered significant if $p<0.05$. Bonferroni correction of $p$-values was applied for multiple comparisons (in particular, comparison of jasplakinolide treated cells, cells implanted in jasplakinolide treated slices and control cells).

\section{Immunohistochemistry}

$4 \mathrm{~d}$ after brain slice preparation the tissue was fixed and pre-embedded in 2\% agar (Carl Roth \# 5210.3)- 2.5\% gelatin (Merck Millipore \# 1040700500; in PBS) without sponge pads and subsequently processed for paraffin embedding. 4-6 $\mu \mathrm{m}$ thick sections were stained according to standard immunohistochemistry protocols. The antibodies used were specific for: glial fibrillary acidic protein (GFAP) (Biolegend \# 644701; diluted 1:500), laminin (Progen Biotech \# 10765; diluted 1:200), myelin basic protein (MBP) (Abcam \# 7349; diluted 1:200) and ionized calcium-binding adapter molecule 1 (Iba1) (Wako \# 019-19,741; diluted $1: 500)$.

\section{Results}

\section{Adult slice cultures retain cytoarchitecture of the brain}

The majority of previous publications utilized brain slices from perinatal donors that show a high degree of resistance to mechanical trauma during slice preparation [23]. However, as high grade gliomas are most common among adult patients, we used adult mice with a fully developed brain, including completed myelination, as donors for the preparation of brain slices. To determine whether the cytoarchitecture is preserved, we performed immunohistochemical staining on adult brain slices embedded 4 days after slicing. We observed that blood vessels and myelinated fiber tracts were present and morphologically intact; astrocytes (GFAP) and microglia (Iba1) were slightly activated within the brain slice, presumably induced by the mechanical trauma of cutting (Fig. 2). In contrast to the survival of astrocytes, microglia and endothelial cells, neuronal survival in brain slices has been reported as a major challenge, especially for slices prepared from adult donors [24]. This is partly attributed to the fact that neuronal cell death is induced by axotomy during the process of tissue slicing. However, the structure of myelinated nerve tracts remains intact and provides the same structural surfaces glioma cells encounter in vivo. Taken together, we confirm that the cytoarchitecture of the adult murine brain is retained in the slices which, thus, represent a suitable three-dimensional matrix to study glioma cell invasion.

\section{Assessment of invasive capacity}

To demonstrate that our ex vivo invasion assay protocol allows a high degree of glioma cell invasion, we manually implanted a panel of DiD labeled human and murine glioma spheroids into adult brain slices that had been adapted to in vitro conditions for 2 days. $48 \mathrm{~h}$ after implantation we fixed the slices and performed confocal 


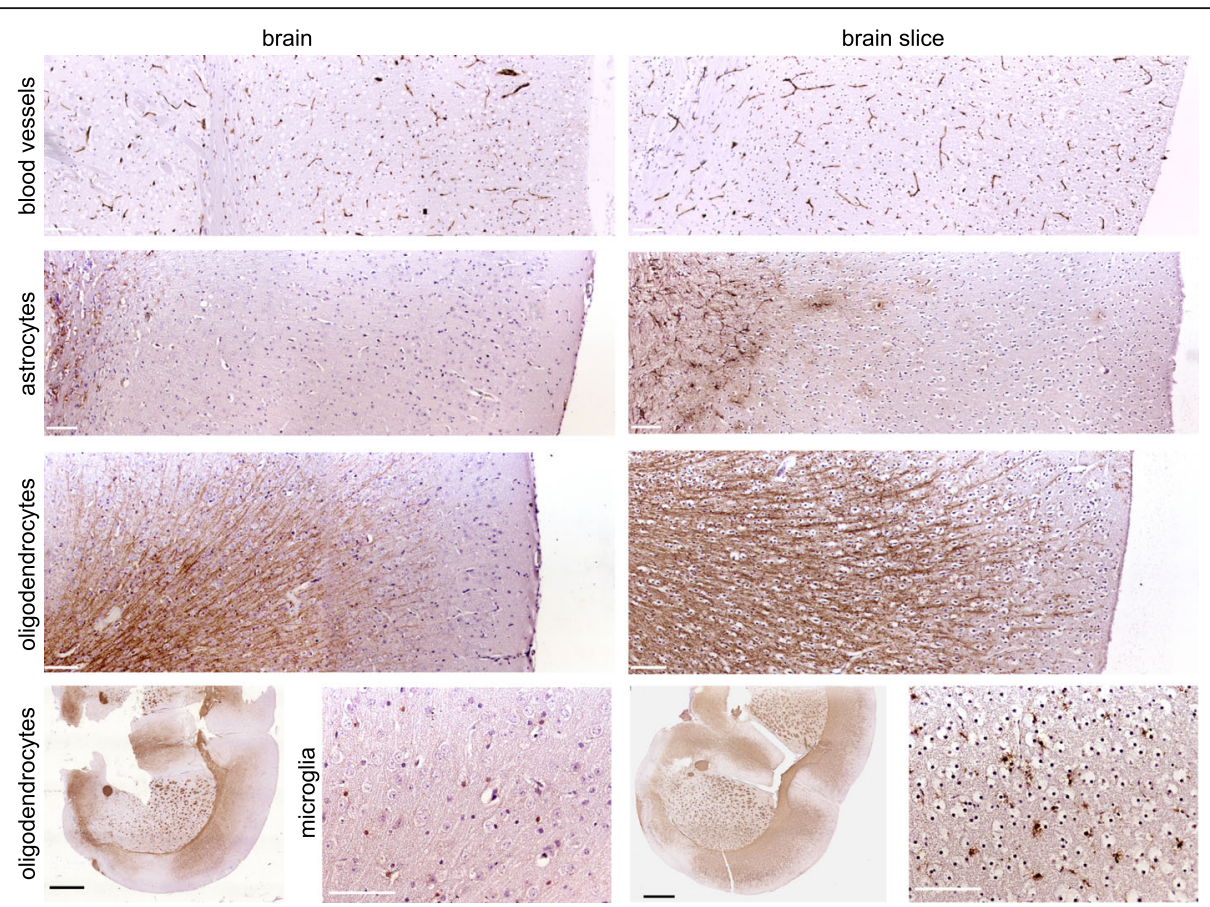

Fig. 2 Organotypic brain slice cultures maintain characteristic features of adult brain tissue. Immunohistochemical staining of 4-6 $\mu$ m sections of adult murine brain slices (prepared from $350 \mu \mathrm{m}$ vibratome sections and cultivated for four days) compared to 4-6 $\mu \mathrm{m}$ sections of whole adult murine brains. Note the comparable patterns of blood vessels, as indicated by laminin, and myelinating oligodendrocytes, as indicated by MBP staining. We observed slight activation of astrocytes (GFAP) and microglia (Iba1) within the brain slice, presumably induced by the mechanical trauma of vibratome cutting. White scale bars $100 \mu \mathrm{m}$, black scale bars $1 \mathrm{~mm}$

imaging. As depicted in Fig. 3 all types of implanted glioma cells invaded strongly into the surrounding tissue. These observations suggest that the invasive capacity of different tumor cells can be reliably assessed and compared using this ex vivo invasion assay protocol.

\section{DiD labeling of tumor cells and optional tissue clearing enables high-contrast imaging}

Although previous studies have used ectopic GFP expression or the carbocyanine dye DiI for membrane labeling and tracing of cell invasion [13, 14, 16], we experienced high autofluorescence of the brain slice and a poor contrast between tissue and tumor cells when imaged with short excitation/emission wavelengths. To reduce this autofluorescent background we used the lipophilic carbocyanine dye DiD, an analog of DiI with markedly redshifted fluorescence excitation and emission spectra. As autofluoresence decreases dramatically at longer wavelengths, DiD labeling resulted in strikingly sharper images compared to DiI (Fig. 4) and is moreover preferable for live cell imaging applications due to reduced photodamaging effects. To further improve epi-fluorescence imaging we cleared the brain tissue according to the SeeDB protocol [17]. As shown in Fig. 5 the quality of epifluorescence images of cleared brain slices was dramatically improved when compared to uncleared tissue. Taken together, our results show that tumor cell labeling with the carbocyanine dye $\mathrm{DiD}$ and brain slice clearing produce high-contrast epi-fluorescence images, representing a comparable alternative to confocal microscopy.

Brain slices prepared from genetically engineered mice are suitable to study the stromal impact on tumor cell invasion The influence of the microenvironment on the invasive behavior of tumor cells has been increasingly recognized. To illustrate that the ex vivo invasion assay enables the study of tumor cell/microenvironment interactions, we used brain slices prepared from a genetically engineered mouse with a global loss of a cell surface protein. We observed a substantial reduction in tumor cell invasion upon spheroid implantation in knockout compared to control wild-type brain slices (Fig. 6a-c). Thus, our results demonstrate the suitability of this assay to study the impact of stromal components on tumor cell invasion by using brain slices from genetically engineered mice.

Heterotypic spheroids allow direct comparison of different cell types and their mutual influence on invasion

To examine the mutual influence of different cell types on their invasive properties, we performed implantations 

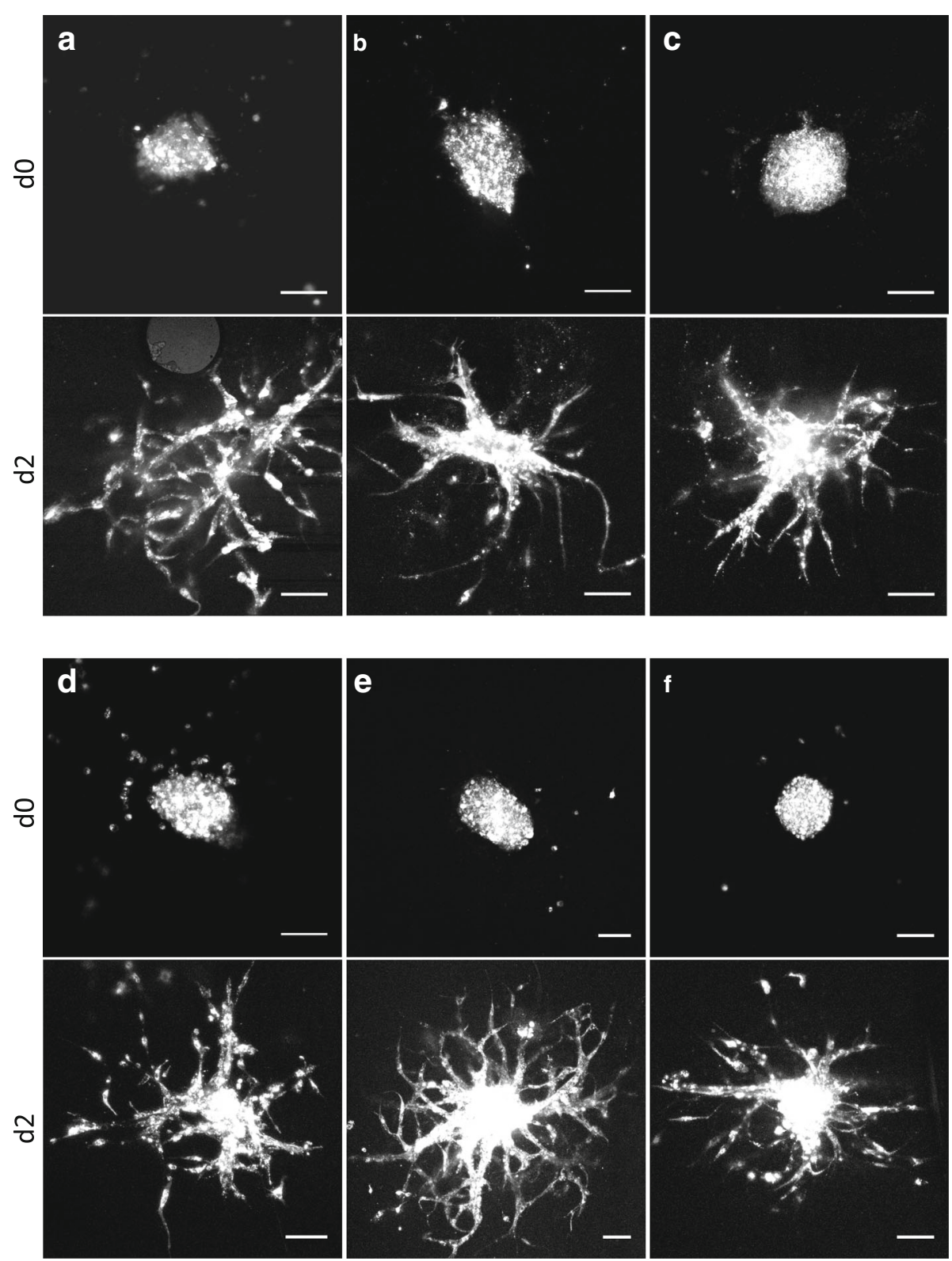

Fig. 3 Improved ex vivo cell invasion assay. Representative confocal images of DiD labeled primary glioma cells (human (a), murine (b)) and established glioma cell lines (murine SMA560 (c) and human LN319 (d), U87MG (e) and U251MG (f)) implanted in adult brain slice cultures. Images were acquired at d0 (top) and d2 (bottom). Scale bars $100 \mu \mathrm{m}$, image quality was optimized by adjustment of brightness, contrast and gamma

of heterotypic spheroids composed of tumor cells and other cell types differentially labeled with fluorescent dyes. In heterotypic spheroids generated from tumor cells and reactive astrocytes, we observed migration of both cell types (Fig. 6d) without identifying one cell type as the leading cell. Similarly, this method allows for the co-cultivation of control and genetically modified tumor cells, i.e. harboring gain or loss of specific proteins, and direct comparison of their invasive behavior.
The ex vivo invasion assay as a tool to identify small molecules affecting invasion

Finally, we evaluated whether the system is suitable for small molecule treatment for the testing of cell invasion modulating compounds. In our experimental setting the drug can be applied either directly to the tumor cells or to the environment. As a proof of principle, we treated tumor spheroids before implantation with jasplakinolide, a known inhibitor of migration $[25,26]$. Previously, an inhibitory effect of $1 \mu \mathrm{M}$ jasplakinolide on actin 


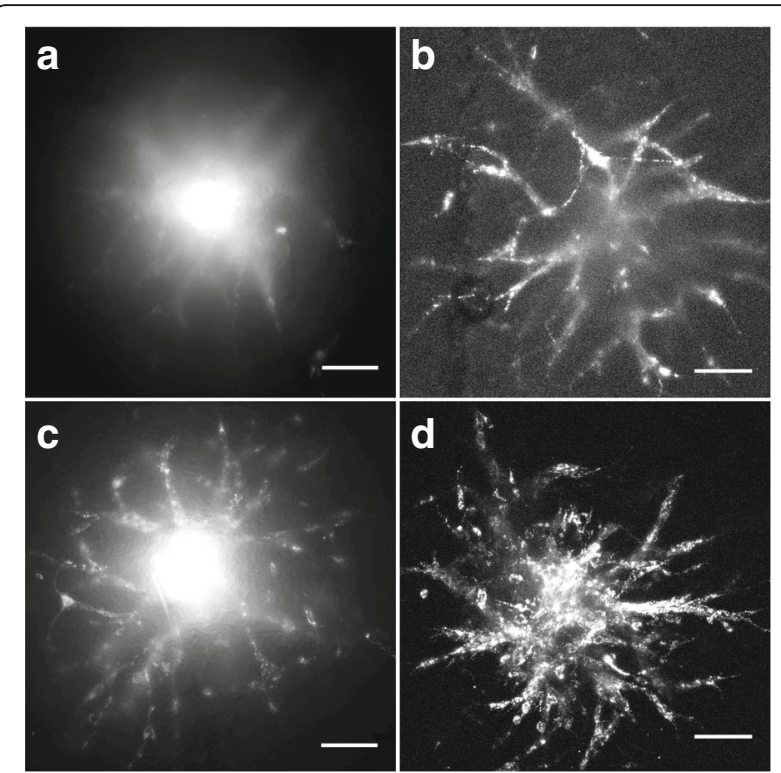

Fig. 4 Improved image quality by DiD labeling and confocal imaging. Representative pictures of epi-fluorescence $(\mathbf{a}, \mathbf{c})$ and confocal microscopy $(\mathbf{b}, \mathbf{d})$. Usage of DiD $(\mathbf{c}, \mathbf{d})$ improves picture quality compared to Dil labeling of SMA560 cells $(\mathbf{a}, \mathbf{b})$. Scale bars $100 \mu \mathrm{m}$ image quality was optimized by adjustment of brightness, contrast and gamma

depolymerization and thus cell migration had been described [26, 27]. Consistent with this, we observed significantly less tumor cell invasion upon tumor cell treatment with $1 \mu \mathrm{M}$ jasplakinolide for $18 \mathrm{~h}$ (Fig. 6e, f, h). Importantly, cell death was not induced under these conditions (Fig. 6i). We obtained similar results when treating the brain slice with $1 \mu \mathrm{M}$ jasplakinolide $18 \mathrm{~h}$ prior to implantation (Fig. 6g). This result highlights the application of our method to drug discovery and preclinical evaluation, by permitting the selection of compounds affecting tumor cell invasion prior to in vivo testing.

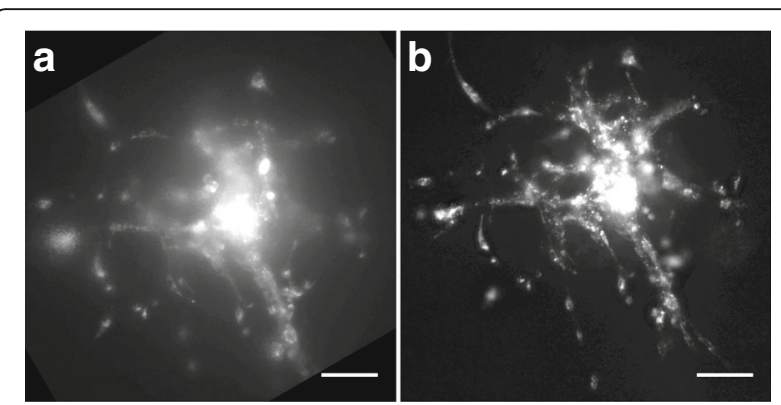

Fig. 5 Improved epi-fluorescence microscopy after tissue clearing. DiD labeled LN319 cells imaged by epi-fluorescence microscope before (a) and after tissue clearing (b). Scale bars $100 \mu \mathrm{m}$, image quality was optimized by adjustment of brightness, contrast and gamma
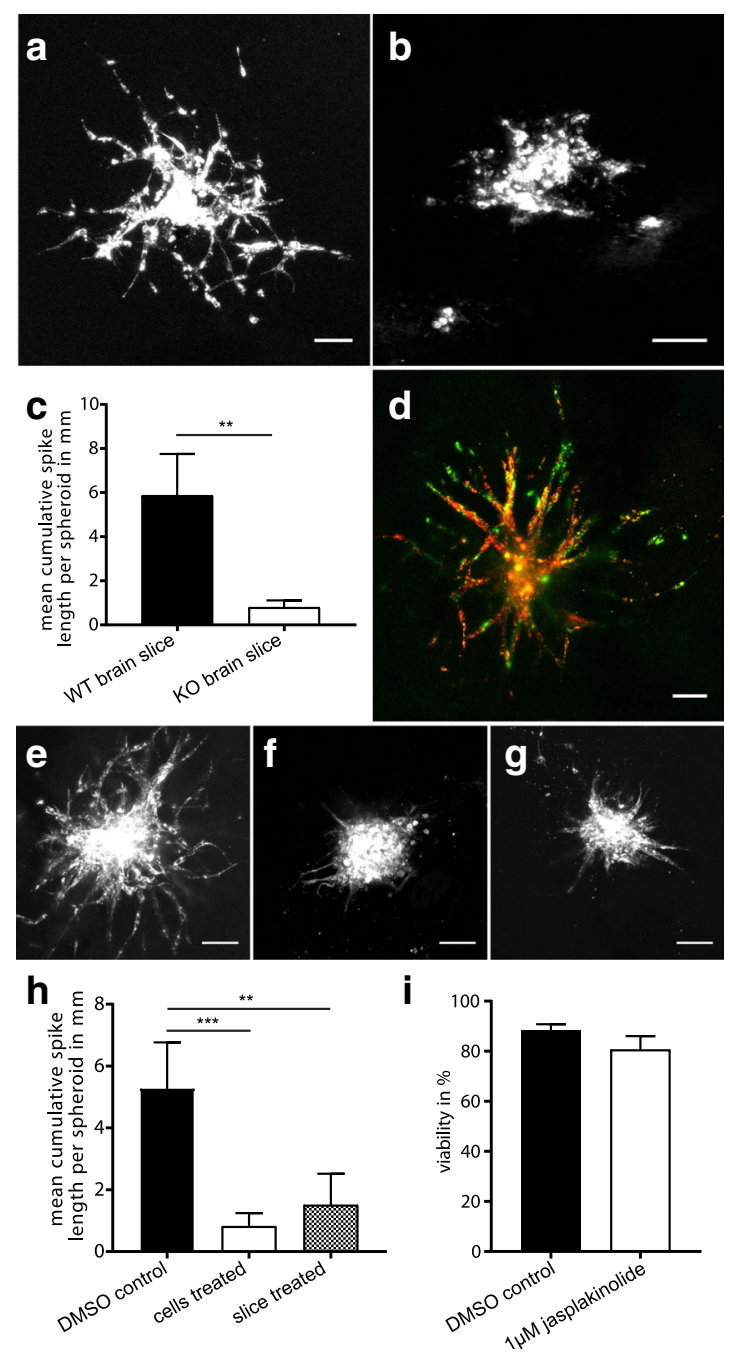

Fig. 6 Multiple applications of the ex vivo invasion assay. Representative confocal images, scale bars $100 \mu \mathrm{m}$, image quality was optimized by adjustment of brightness, contrast and gamma. a-c Brain slices of genetically modified mice as a tool to modulate the microenvironment. Human glioma cell line LN319 implanted in a wild-type and b knockout brain slices. The deletion of a specific cell surface protein in the microenvironment significantly inhibits tumor cell invasion as quantified in (c). Error bars represent 95\% confidence interval, (A) $n=7$; (B) $n=5$; ** $p<0.001$, Welch's t-test. $\mathbf{d}$ Co-implantation of different cell types. Reactive astrocytes (pseudocolored in green, Dil labeled) and the murine glioma cell line SMA560 (red, DiD labeled) were co-cultured in a ratio of 3:2 as heterotypic multicellular spheroids and implanted in a wild-type brain slice. e-i The ex vivo invasion assay as a tool to identify invasion modulating compounds. 500 SMA560 glioblastoma cells were seeded per well to induce spheroid growth. $18 \mathrm{~h}$ prior to implantation, spheroids or brain slices were treated with $1 \mu \mathrm{M}$ jasplakinolide. $24 \mathrm{~h}$ after implantation, brain slices were fixed and imaged by confocal microscopy. In contrast to e the highly invasive control-treated SMA560, $\mathbf{f}$ the treatment of the tumor cells or $\mathbf{g}$ brain slices with jasplakinolide significantly reduced their ability to invade without inducing cell death as examined by trypan blue staining (i). $\mathbf{h}$ Quantification of invasion. Error bars show 95\% confidence interval, e $n=12 ; \mathbf{f}, \mathbf{g ~ n}=7 ; \mathbf{i} n=3 ;{ }^{* *} p<0.001 ;{ }^{* * *} p \leq 0.0001$; Welch's t-test, $p$-values Bonferroni corrected 


\section{Discussion}

In the past decades tumor cell invasion has primarily been assessed by inexpensive and rapid two-dimensional assays. However, these cell culture models are very limited in their power to accurately predict the effect of proteins or small molecules on cell invasion in vivo, probably due to functional differences of proteins between two- and three-dimensional migration and the absence of environmental influences [28]. Although animal models are thought to represent the most reliable method of investigating cell invasion, they involve not only high cost but also ethical and technical concerns. The laborious and time inefficient application of mouse models is especially disadvantageous for co-clinical and personalized treatment studies that rely on a rapid read out and the exclusion of falsepositive candidate compounds identified by conventional two-dimensional assays [29]. This has resulted in attempts to bridge the gap between over-simplified cell culture approaches and the more meaningful, but inefficient, in vivo models with reproducible ex vivo techniques. The current state of the art to mimic the natural environment of glioma cells are organotypic brain slice cultures that can be cultivated ex vivo for several days to weeks without considerable loss of their cytoarchitecture. Retaining their physiological structure, brain slices provide an optimal three-dimensional matrix for ex vivo invasion assays. To our knowledge, mostly perinatal donors have been used for the preparation of organotypic brain slices due to their high mechanical and ischemic resistance. However, in rodents the ECM is substantially remodeled starting from 2 weeks after birth. This remodeled and thus significantly firmer ECM is subsequently maintained throughout adulthood [30]. Similarly, myelination of nerve fibers occurs predominantly postnatally and can be extended to adulthood [31]. Hence, absent or incomplete myelination and the immature and loose extracellular matrix are profound differences between neonatal and mature adult brain tissue. In order to reflect the age related disease of adult glioma, we decided to use adult brain slices that exhibit a mature myelination pattern and ECM composition.

While there are previous reports that use organotypic brain slices for tumor cell invasion assessment, we still lack a simple, standardized, and reproducible protocol that allows its application in basic and preclinical research. Various approaches for the co-cultivation of tumor cells and slices and the measurement of cell invasion have been published. For brain metastasis research, tumor cells have been seeded in a matrigel plug adjacent to the brain slice in order to investigate interactions between cancer and glial cells by fluorescence microscopy [32]. However, this model is unsatisfactory when used to examine the invasion of primary brain tumor cells, as they arise and migrate within the brain tissue and moreover do not encounter an environment comparable to matrigel. Other publications have developed this approach by seeding single cells on top of the slices [33]. However, we see disadvantages in this technique, as it is disturbed by the diffusion of single cells after seeding and requires life cell imaging, preferably by an upright confocal microscope, which is not universally available. Moreover, we have observed reduced invasion when the tumor cells were seeded on top instead of within the tissue, as the cells migrate on the slice surface instead of efficiently penetrating the tissue. In contrast, we implant tumor cells as spheroids within the tissue in order to position them at a specific location of the brain slice and to provide a comparable starting point for the assay. Indeed, other publications have reported this approach; however, they show a low grade of invasion that does not reflect the aggressive infiltration observed in patients $[13,15,16]$. Following our protocol, we could observe strong invasion in a panel of human and murine glioma cell lines as well as primary cells. Moreover, we could improve the imaging quality of implanted tumor cells using $\mathrm{DiD}$, which drastically reduces autofluorescence compared to the commonly applied DiI. Epi-fluorescence microscopy was further optimized by tissue clearing according to the SeeDB protocol [17]. Thus, we have generated an optimized and standardized protocol that acts as the basis of a set of functional applications, some of which we describe here. The possibility of specifically manipulating either one or both compartments involved in tumor cell migration, the microenvironment and the tumor cells themselves, and to monitor the consequences of the manipulation on tumor cell invasion is a major advantage of this improved protocol. Here, we demonstrate the flexibility of the protocol by manipulating the microenvironment with exogenous small molecule treatment or using genetically modified mice as donors for the organotypic brain slice cultures. This allows the investigation of the effects of global inactivation of a gene or gene product of interest, as well as cell type-specific deletion or overexpression, depending on the mouse model used. In addition, this assay is suitable for direct manipulation of the tumor cells themselves by gainor loss-of-function approaches. Consequently, the combination of both strategies is a powerful tool i) to identify critical factors in tumor cells and their putative interaction partners in the tumor microenvironment and ii) to dissect their mode of action in tumor cell invasion, ultimately yielding potential novel targets for brain tumor therapy.

\section{Conclusion}

As growing evidence suggests the tumor microenvironment as a key factor in tumor cell invasion, we optimized an ex vivo invasion assay that can reliably and quantitatively measure glioma cell movement in an environment that more accurately recapitulates the 
physiological state in vivo. The functional applications described demonstrate the power and versatility of this method as an advance over previous work, and will encourage the use of more relevant models than traditional two-dimensional migration assays.

\section{Abbreviations}

DiD: 1,1'-dioctadecyl-3,3,3',3'- tetramethylindodicarbocyanine,

4-chlorobenzenesulfonate salt; Dil: 1,1'-dioctadecyl-3,3,3',3'-

tetramethylindocarbocyanine; DMEM: Dulbecco's Modified Eagle Medium; DMSO: Dimethyl sulfoxide; ECM: extracellular matrix; EGF: Epidermal growth factor; FGFb: Basic fibroblast growth factor; GFAP: glial fibrillary acidic protein; GFP: Green fluorescent protein; HEPES: 4-(2-hydroxyethyl)-1piperazineethanesulfonic acid buffer; Iba1: ionized calcium-binding adapter molecule 1; MBP: myelin basic protein; MEM: Minimum Essential Medium: PBS: Phosphate Buffered Saline

\section{Acknowledgements}

We thank Dr. Annette Kopp-Schneider for advice on statistical analysis, Dr. Michael Fletcher for proofreading the manuscript, Sabrina Lohr for technical support and Tobias Schmidt for helpful discussions. MM would like to thank the Luxembourg National Research Fond (FNR) for the support (FNR PEARL P16/BM/11192868 grant)

\section{Funding}

This work was supported by the Helmholtz Alliance Preclinical Comprehensive Cancer Center (to PA).

\section{Availability of data and materials}

The datasets used and/or analysed during the current study are available from the corresponding author on reasonable request.

\section{Authors' contributions}

TE and HP designed the study; TE and BC conducted the experiments; JS helped with brain slice preparation; MM provided primary human brain tumor material; data was interpreted and discussed by TE, BC, JS, MM, PA, HP; the manuscript was written by TE, PA and HP. All authors have read and approved the final version of this manuscript.

\section{Ethics approval and consent to participate}

Experiments involving human patient material were performed in accordance with the Declaration of Helsinki and were approved by the ethics committee of the University Cancer Center Frankfurt, Project number SNO_01_13. All animal experiments were approved by the responsible authority for animal experiments (Regierungspraesidium Karlsruhe, Germany) and performed in conformity with the German Law for Animal Protection.

\section{Consent for publication}

Not applicable

\section{Competing interests}

The authors declare that they have no competing interests.

\section{Publisher's Note}

Springer Nature remains neutral with regard to jurisdictional claims in published maps and institutional affiliations.

\section{Author details}

'Division of Signal Transduction and Growth Control, DKFZ/ZMBH Alliance, Heidelberg, Germany. ${ }^{2}$ Functional Neuroanatomy, University of Heidelberg, Heidelberg, Germany. ${ }^{3}$ Institute of Neurology (Edinger-Institute), University Hospital Frankfurt, Goethe University, Frankfurt, Germany. ${ }^{4}$ Luxembourg Centre of Neuropathology (LCNP), Dudelange, Luxembourg. ${ }^{5}$ Laboratoire National de Santé, Dudelange, Luxembourg. ${ }^{6}$ Luxembourg Centre for Systems Biomedicine (LCSB), University of Luxembourg, Esch-sur-Alzette, Luxembourg. ${ }^{7}$ Department of Oncology, NORLUX Neuro-Oncology Laboratory, Luxembourg Institute of Health (L.I.H.), Strassen, Luxembourg. ${ }^{8}$ Present address: Translational Program, Hopp Children's Cancer Center at NCT Heidelberg (KiTZ), University Hospital and DKFZ Heidelberg, Heidelberg,
Germany. ${ }^{9}$ Present address: Clinical Cooperation Unit Pediatric Oncology, DKFZ, Heidelberg, Germany. ${ }^{10}$ German Consortium for Translational Cancer Research (DKTK), Heidelberg, Germany.

Received: 6 June 2017 Accepted: 18 January 2018

Published online: 30 January 2018

\section{References}

1. Chaudhry NS, Shah AH, Ferraro N, Snelling BM, Bregy A, Madhavan K, Komotar RJ. Predictors of long-term survival in patients with glioblastoma multiforme: advancements from the last quarter century. Cancer Investig. 2013;31(5):287-308. https://doi.org/10.3109/07357907.2013.789899.

2. Johnson DR, O'Neill BP. Glioblastoma survival in the United States before and during the temozolomide era. J Neuro-Oncol. 2012;107(2):359-64. https://doi.org/10.1007/s11060-011-0749-4.

3. Krex D, Klink B, Hartmann C, von Deimling A, Pietsch T, Simon M, Sabel M Steinbach JP, Heese O, Reifenberger G, et al. long-term survival with glioblastoma multiforme. Brain. 2007;130(Pt 10):2596-606. https://doi.org/10. 1093/brain/awm204

4. Paw I, Carpenter RC, Watabe K, Debinski W, Lo HW. Mechanisms regulating glioma invasion. Cancer Lett. 2015;362(1):1-7. https://doi.org/10.1016/j. canlet.2015.03.015.

5. Xie Q, Mittal S, Berens ME: Targeting adaptive glioblastoma: an overview of proliferation and invasion. Neuro-Oncology 2014, 16(12):1575-1584.

6. Khatau SB, Bloom RJ, Bajpai S, Razafsky D, Zang S, Giri A, Wu PH, Marchand J, Celedon A, Hale CM, et al. The distinct roles of the nucleus and nucleuscytoskeleton connections in three-dimensional cell migration. Sci Rep. 2012;2:488.

7. Madsen CD, Hooper S, Tozluoglu M, Bruckbauer A, Fletcher G, Erler JT, bates PA, Thompson B, Sahai E. STRIPAK components determine mode of cancer cell migration and metastasis. Nat Cell Biol. 2015;17(1):68-80. https://doi.org/10.1038/ncb3083.

8. Skau CT, Fischer RS, Gurel P, Thiam HR, Tubbs A, Baird MA, Davidson MW, Piel M, Alushin GM, Nussenzweig A, et al. FMN2 makes perinuclear actin to protect nuclei during confined migration and promote metastasis. Cell. 2016;167(6):1571-85 e1518. https://doi.org/10.1016/j.cell.2016.10.023.

9. Joyce JA, Pollard JW. Microenvironmental regulation of metastasis. Nat Rev Cancer. 2009;9(4):239-52. https://doi.org/10.1038/nrc2618.

10. Cuddapah VA, Robel S, Watkins S, Sontheimer H. A neurocentric perspective on glioma invasion. Nat Rev Neurosci. 2014;15(7):455-65. https://doi.org/10. 1038/nrn3765.

11. Huang Y, Williams JC, Johnson SM. Brain slice on a chip: opportunities and challenges of applying microfluidic technology to intact tissues. Lab Chip. 2012;12(12):2103-17. https://doi.org/10.1039/c2lc21142d.

12. Lossi L, Alasia S, Salio C, Merighi A. Cell death and proliferation in acute slices and organotypic cultures of mammalian CNS. Prog Neurobiol. 2009;88(4):221-45. https://doi.org/10.1016/j.pneurobio.2009.01.002.

13. Aaberg-Jessen C, Norregaard A, Christensen K, Pedersen CB, Andersen C, Kristensen BW. Invasion of primary glioma- and cell line-derived spheroids implanted into corticostriatal slice cultures. Int J Clin Exp Patho. 2013:6(4):546-60.

14. Jung S, Kim HW, Lee JH, Kang SS, Rhu HH, Jeong Yl, Yang SY, Chung HY, Bae CS, Choi $C$, et al. Brain tumor invasion model system using organotypic brain-slice culture as an alternative to in vivo model. J Cancer Res Clin Oncol. 2002;128(9):469-76. https://doi.org/10.1007/s00432-002-0366-x.

15. Petterson SA, Jakobsen IP, Jensen SS, Aaberg-Jessen C, Nielsen M, Johansen J, Kristensen BW. Implantation of glioblastoma spheroids into organotypic brain slice cultures as a model for investigating effects of irradiation: a proof of concept. Int J Clin Exp Patho. 2016:9(4):4816-23.

16. Xu WL, Wang Y, Wu J, Li GY. Quantitative analysis of U251MG human glioma cells invasion in organotypic brain slice co-cultures. Eur Rev Med Pharmaco. 2016:20(11):2221-9.

17. Ke MT, Fujimoto S, Imai T. SeeDB: a simple and morphology-preserving optical clearing agent for neuronal circuit reconstruction. Nat Neurosci. 2013;16(8):1154-U1246. https://doi.org/10.1038/nn.3447.

18. Schneider CA, Rasband WS, Eliceiri KW. NIH image to ImageJ: 25 years of image analysis. Nat Methods. 2012;9(7):671-5. https://doi.org/10.1038/ nmeth.2089.

19. Heiss M, Hellstrom M, Kalen M, May T, Weber H, Hecker M, Augustin HG, Korff T. Endothelial cell spheroids as a versatile tool to study angiogenesis in vitro. FASEB J. 2015;29(7):3076-84. https://doi.org/10.1096/fj.14-267633. 
20. Korff T, Augustin HG. tensional forces in fibrillar extracellular matrices control directional capillary sprouting. J Cell Sci. 1999;112(Pt 19):3249-58.

21. Stahl A, Wu X, Wenger A, Klagsbrun M, Kurschat P. Endothelial progenitor cell sprouting in spheroid cultures is resistant to inhibition by osteoblasts: a model for bone replacement grafts. FEBS Lett. 2005;579(24):5338-42. https://doi.org/10.1016/j.febslet.2005.09.005.

22. Weber H, Claffey J, Hogan M, Pampillon C, Tacke M. Analyses of Titanocenes in the spheroid-based cellular angiogenesis assay. Toxicol in Vitro. 2008;22(2):531-4. https://doi.org/10.1016/j.tiv.2007.09.014.

23. Cho S, Wood A, Bowby MR. Brain slices as models for neurodegenerative disease and screening platforms to identify novel therapeutics. Curr Neuropharmacol. 2007;5(1):19-33. https://doi.org/10.2174/157015907780077105.

24. Humpel C. Organotypic brain slice cultures: a review. Neuroscience. 2015;305:86-98. https://doi.org/10.1016/j.neuroscience.2015.07.086.

25. Ivkovic S, Beadle C, Noticewala S, Massey SC, Swanson KR, Toro LN, Bresnick AR, Canoll P, Rosenfeld SS. Direct inhibition of myosin II effectively blocks glioma invasion in the presence of multiple motogens. Mol Biol Cell. 2012;23(4):533-42.

26. Ponti A, Machacek M, Gupton SL, Waterman-Storer CM, Danuser G. Two distinct actin networks drive the protrusion of migrating cells. Science. 2004;305(5691):1782-6. https://doi.org/10.1126/science.1100533.

27. Mseka T, Cramer LP. Actin depolymerization-based force retracts the cell rear in polarizing and migrating cells. Curr Biol. 2011;21(24):2085-91. https://doi.org/10.1016/j.cub.2011.11.006.

28. Pampaloni F, Reynaud EG, Stelzer EH. The third dimension bridges the gap between cell culture and live tissue. Nat Rev Mol Cell Biol. 2007;8(10):839-45. https:/doi.org/10.1038/nrm2236.

29. Jensen SS, Petterson SA, Halle B, Aaberg-Jessen C, Kristensen BW. effects of the lysosomal destabilizing drug siramesine on glioblastoma in vitro and in vivo. BMC Cancer. 2017;17(1):178.

30. Zimmermann DR, Dours-Zimmermann MT. Extracellular matrix of the central nervous system: from neglect to challenge. Histochem Cell Biol. 2008;130(4):635-53. https://doi.org/10.1007/s00418-008-0485-9.

31. Semple BD, Blomgren K, Gimlin K, Ferriero DM, Noble-Haeusslein LJ. Brain development in rodents and humans: identifying benchmarks of maturation and vulnerability to injury across species. Prog Neurobiol. 2013;106-107:1-16. https:/doi.org/10.1016/j.pneurobio.2013.04.001.

32. Chuang HN, Lohaus R, Hanisch UK, Binder C, Dehghani F, Pukrop T. Coculture system with an organotypic brain slice and 3D spheroid of carcinoma cells. J Vis Exp. 2013;(80) https://doi.org/10.3791/50881.

33. Chadwick E, Yang DP, Filbin MG, Mazzola E, Sun Y, Behar O, Pazyra-Murphy MF, Goumnerova L, Ligon KL, Stiles CD, et al. A brain tumor/Organotypic slice co-culture system for studying tumor microenvironment and targeted drug therapies. J Vis Exp. 2015;105:e53304.

\section{Submit your next manuscript to BioMed Central and we will help you at every step:}

- We accept pre-submission inquiries

- Our selector tool helps you to find the most relevant journal

- We provide round the clock customer support

- Convenient online submission

- Thorough peer review

- Inclusion in PubMed and all major indexing services

- Maximum visibility for your research

Submit your manuscript at www.biomedcentral.com/submit

Biomed Central 